\title{
Facilitating Image and Document Retrieval Using Image Content and Querying Keyword
}

\author{
MR Narasinga Rao ${ }^{1}, \mathrm{~J} \mathrm{Srinija}^{2}$, P Krishna Chaitanya ${ }^{3}$, Y Chanukya ${ }^{4}$, \\ T Sabhareesh ${ }^{5}$ \\ Department Computer Science and Engineering, Koneru Lakshmaiah University \\ Vaddeswaram, Andhra Pradesh 522502, India \\ e-mail: ramanarasingarao@kluniversity.in ${ }^{1}$, srinija94@gmail.com ${ }^{2}$, \\ chaitanyakrishna414@gmail.com ${ }^{3}$, chanukyayanduru@gmail.com ${ }^{4}$, sabhareesh551@gmail.com $^{5}$
}

\begin{abstract}
Evolution of ubiquitous computing in the areas of personal computing technology has produced staggeringly large data It is difficult to search mainly the image data by understanding users objective only by keywords and phrases and this leads to uncertain outcomes. For producing these outcomes effectively, this paper introduces a new approach to the problem of image learning to enable search engines to learn about visual content over time based on user feedback through one click activity and images from a pool recovered by text based query are re-ranked depending on both visual and text based query. Content Based Image Retrieval (CBIR) techniques are used for accessing semantically-relevant images from an image data source depending on automatically-derived image functions for features like Geometric moments, Global histogram, Color Moments, Local histogram. Documents can also be retrieved using the text based query by the user.
\end{abstract}

Keywords: Image re-ranking, Content Based Image Retrieval, Geometric moments, Global histogram, Local histogram, Color Moments

\section{Introduction}

One of the main problems highlighted was difficulty in locating a desired image in a large and varied collection while it is feasible to identify a desired image from a small collection by browsing. More effective techniques are needed with database containing thousands of items. Journalists collecting photographs of a particular type of event, designers searching for materials with a particular color or texture need some access by image content.

Users type query keywords to find a certain type of images. The search engine retrieves thousands of images ranked by the keywords extracted from the surrounding text. It is known that text-based image search sometimes results in ambiguity of query keywords. The keywords provided by users tend to be short.

Kinds of query users are likely to put to an image database depends on why users search for images, what use they make of them, and how they judge the utility of the images they retrieve. Potentially, images have many types of attribute which could be used for retrieving purpose and this includes

- The images having particular combination of color, texture or shape features (e.g. green stars);

- The organization of specific types of object (e.g. chairs around a table);

- The representation of a particular type of event (e.g. a football match);

- The existence of named individuals, locations, or events (e.g. the Queen greeting a crowd);

- Particular emotions one might associate with the image (e.g. happiness);

- Metadata implies who, where and when the image is created.

Each above listed query type (with the exception of the last) denotes a higher level of abstraction than its predecessor, and each is more difficult to answer without refering external knowledge. This leads naturally to classify query types into three levels according to the increasing complexity.

Level 1 comprises retrieval by primitive features. Level 1 type of retrieval uses features that are both objective, and are derivable directly from external knowledge.

Level 2 encloses retrieval by logical features, having some degree of logical inference about the uniqueness of the objects depicted in the image. It can be divided into: 
1. Retrieval of objects belonging to a given type (e.g. "find images of a metro bus")

2. Retrieval of particular objects or persons ("find images of the greatest cricket player").

Level 3 infers about retrieving abstract attributes, which involves a significant amount of high-level reasoning about the tenacity of the objects or scenes depicted. Again, this level 3 can be sub divided into:

1. Retrieval of type of events (e.g. "find images of playing games").

2. Retrieval of images with emotional or religious significance ("find a images depicting anxiety").

To fix the indecisiveness, more information is to be used to catch users search objective. One way is text-based keyword and key phrase development, which makes the textual information of the question more specific.

\section{CBIR TECHNIQUES:}

Content Based Image Retrieval (CBIR) [4], [5], [8], [9], is a set of techniques for accessing semantically-relevant images from a images data source depending on automatically-derived image features.

Color Retrieval: Several methods for retrieving images on the basis of color similarity have been described in the literature, where most are variations on the same basic idea. Each image that is added to the collection is analyzed to compute a color histogram which shows the proportion of pixels of each color within the image. Then color histogram belonging to each image is then stored in the database. At search time, user can submit an example image from which a color histogram is calculated. Matching process then retrieves those images whose color histograms match those of the query most closely.

Mean Color: Image search is done from calculating the RGB values of an image. Pixel Color Information refers to $R, G, B(R E D, G R E E N, B L U E)$.

Sum of that component for all pixels

Mean component $(\mathrm{R}, \mathrm{G}$ or $\mathrm{B})=\frac{\text { Number of pixels }}{\mathrm{S}}$

In the CBIR system, we used global color histograms to extract the color features of images. We use the HSV (Hue, Saturation, and Value) color space for the simple transformation from the RGB (Red, Green, Blue) color space, in which images are commonly represented.Quantization of HSV is done into 108 bins by using uniform quantization (12 for $\mathrm{H}, 3$ for $\mathrm{S}$, and 3 for $\mathrm{V}$ ). Since Hue $(H)$ has more importance in human visual system than saturation $(S)$ and value $(V)$, it is reasonable to assign bins in the histogram to Hue more than the other components. It is easy to generate the histograms of color images using the selected quantized color space.

Shape retrieval: A number of features characteristic of object shape (but independent of size or orientation) are computed for every object identified within each stored image. Queries are answered by computing the same set of features for the query image, and retrieving the images in the database whose features most closely match those of the query image. Two types of shape feature commonly used are aspect ratio, circularity and moment invariants and a regionbased retrieval program [7] is applicable for image segmentation to break down a images into areas, which match to things. The object-level reflection is designed to be close to the understanding of the human visible program (HVS). Since the retrieval program recognizes what things are in the images, it is easier for the program to identify similar things at different places and with different orientations and dimensions. 


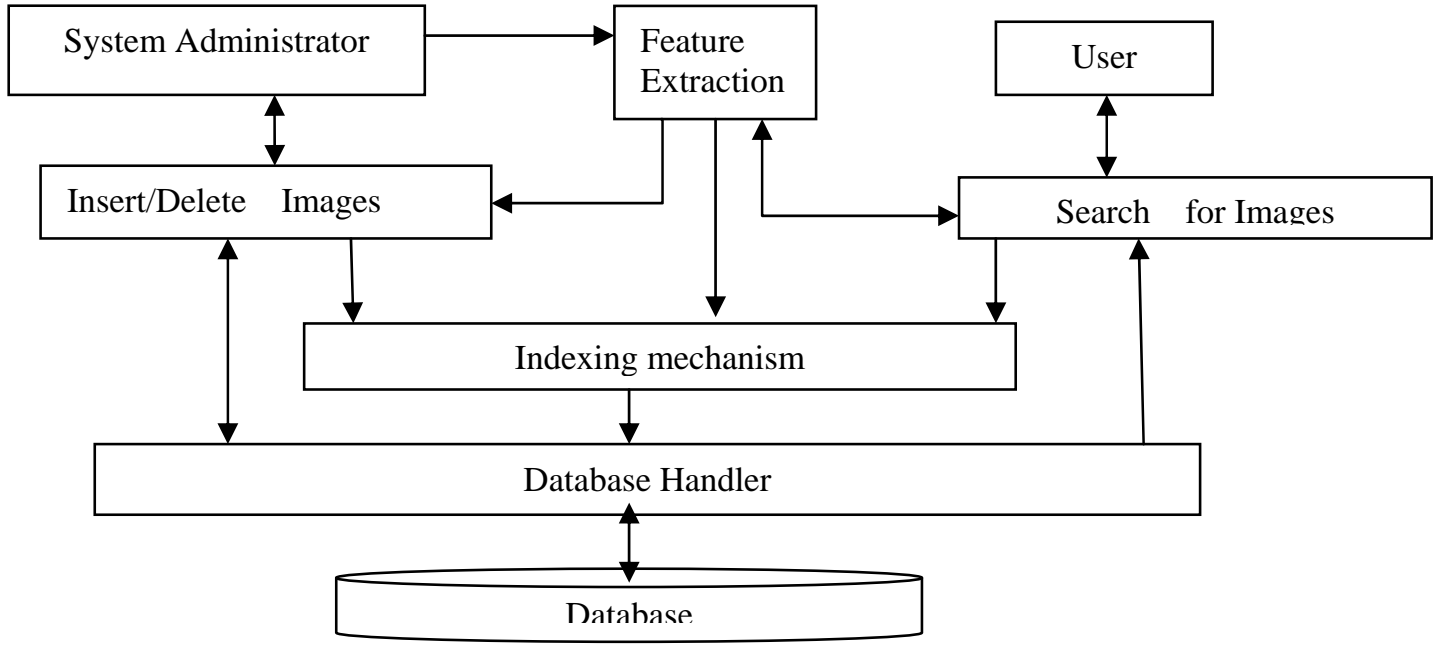

Figure 1. CBIR Architecture

TRADITIONAL APPROACH:

In Existing System, [1] internet driven image search engines use only keywords as queries. Text-based image searching suffers from the ambiguity of query keywords. It uses Adaptive Weight Schema to capture user Intent and re rank results based on it.

Pre Operations: Adaptive Weight Schema categorized into pre-operations which has two sub categories

Query Categorization : The query categories we considered are: General Object, Object having Simple Background, Scenery Images, Portrait, and People.

Feature Fusion: Each query category, requires a pre-training Dynamic Operations: Keyword Expansion is a dynamic operation because it has to be performed while retrieving results for a search. After the top $\mathrm{k}$ images most similar to the query image are found according to the visual similarity metric, words from their textual descriptions are ranked after extraction. The top $\mathrm{m}$ words are reserved as candidates for Visual query expansion which is also a dynamic operation to continuously alter the results based on user validations. Image Pool Expansion is a dynamic operation which executes the queries of Visual Query Expansion continously and obtain results. Based on these Pre and dynamic operations, we have customized results of their choice based on their intent.
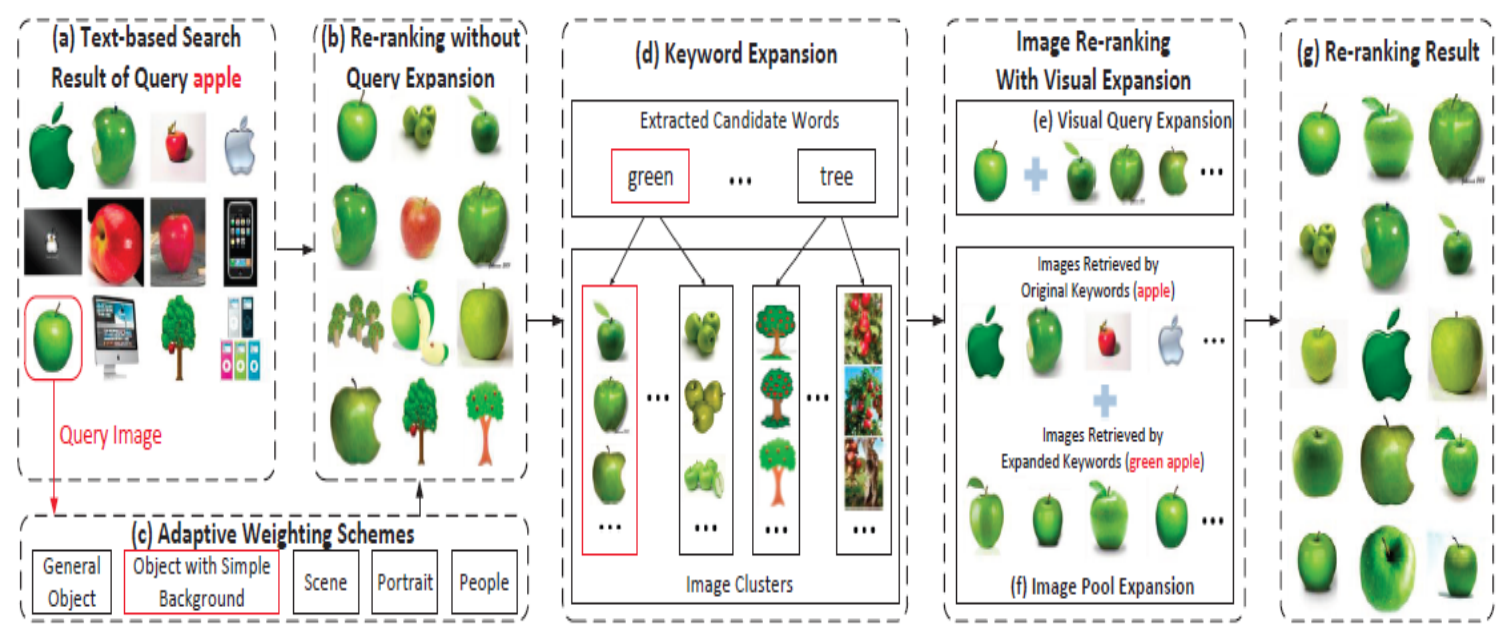

Courtesy from [1] Figure 2. Image retrieval approaches based on process generation with suitable examples 
The customer objective is first approximately taken by identifying the query image into one of the rough semantic groups and choosing a proper bodyweight schema accordingly. Intention specific bodyweight schema is suggested to merge visible functions and to estimate visible likeness flexible to query images. Without additional human reviews, textual and visible expansions are incorporated to catch customer objective. Extended search phrases are used to improve positive example images and also expand the images pool to include more relevant images.

\section{Method}

In the proposed system, there is an option to register and login to the system to search for the required information. In this system, user will retrieve the images by first querying using keyword and then by making the user to click the image desired form the retrieved pool of images, similar images are re ranked and retrieved [2], [3].This is done by comparing some of the features of the images and then retrieving the similar images on a feature basis.

Features considered in our system are:

1. Average RGB (Red, Green, Blue)

2. Color moments

3. Global color Histogram

4. Local Color Histogram

5. Geometric moments

\section{Average RGB}

Images similar to the image selected by the user are retrieved by using average RGB feature. We can perform comparison and retrieve the similar images by using the color and spatial information of the images [12], [13].

\section{Color Moments}

Color moments are measures used to differentiate images based on their features of color. We can measure the color similarity between images by calculating these Color Moments. These values for the images are compared to the values of images in the image pool retrieved by the user for the purpose of the feature based image retrieval.

We used Earth movers distance algorithm [10] [11] for this purpose.

\section{Color Histogram}

Color Histogram represents the distribution of colors in an image. Color histogram represents the image but from another perspective by counting the similar pixels and storing it in bins in order to describe the number of pixels in each range of colors (or bin) independently.

\section{Color Histogram is divided into}

- Global Color Histogram (GCH).

- Local Color Histogram (LCH).

\section{Global Color Histogram}

$\mathrm{GCH}$ is the most known color histogram used to detect similar images. By using this feature also similar image to the selected image are retrieved from the image pool.

\section{Local Color histogram}

$\mathrm{LCH}$ includes information about colors distribution in different regions. It is similar to $\mathrm{GCH}$ but we divide image into number of blocks. We used Multi-Layer Rotation Invariant algorithm [14] to retrieve the similar images from the image pool

\section{Geometric Moments}

Geometric moment is defined as a certain particular weighted average (moment) of the image pixel intensities or a function of such moments, chosen to have some attractive property or interpretation. These are useful to describe segmented objects. Properties which are found using image moments includes area (or total intensity), centroid, and also the information about its orientation. We can also retrieve similar images based on these features [6], [15], [16]. 
Retrieving Documents: Along with the images, it is are also necessary To retrieve the relevant documents needed by the user. We will retrieve the documents required by the user by making the user to query for the required documents. Documents can be retrieved in two ways.

1. Document frequency method: Here the documents are retrieved along with the publication dates and number of time sit is referred by having the number of hits.

2. Inverse Document frequency method: In this method, documents are retrieved from the database a with the keywords are highlighted in the documents in different color.

Algorithm

Step 1.Start

Step2.If the user does not want to login to the system

A) Go to Step 4 and Continue searching the web as guest else

B) Click the link named "login"

Step3: If the user clicks "login"

A) If the user is new to the system then click "register" system.

i) Enter the details of the user and again enter the login credentials to logon to the else

B) Enter the login credentials and click "Submit"

Step 4: User is directed to the search page related to images.

search"

A) If the user wants to refer to the documents then click on the "go to documents

number of hits.

i) enter the query keyword to search for the required document

ii) Required documents will be displayed along with its publishing dates and else

B) User enters the keyword to search for the required image

i) Pool of images are retrieved according to the query keyword.

ii) User selects the image to get the relevant images of it.

iii) Relevant images similar to the selected image are retrieved by the system according to the features

Step5: Stop

We can retrieve the images according to the feature separately so that user can understand which image to select as it will be clear for the user to examine each feature of the images and select the required image. As there is a facility to login to the system, system stores the user interactions according to the name of the user which makes it easy to the administrator to check each user's history when needed. We can also retrieve the exact documents required by the user through this system.

\section{Experimental Results} follows:

In this area consider the features of the conventional and suggested techniques as Image information source and execution environment: The database was used to access the assessment of the images recovery process. It includes 1000 images, a part of the Corel information source, which has been personally chosen to be a information source of 10 sessions of 100 images each. The images are of dimension $384 \times 256$ or $256 \times 384$ p. This information source was substantially used to test many CBIR systems because dimension, information source and the accessibility to category information allows for efficiency assessment.

\section{Evaluation with Images}

We arbitrarily chosen 20 images as concerns from each of the 10 semantic sessions in the information source. For each question, the perfection of the recovery at each level is acquired by progressively improving the number of recovered images.

Facilitating Image and Document Retrieval Using Image Content and ... (M.R. Narasinga Rao) 


\section{Evaluation with Document}

In this section we process to develop efficient search mechanism for document annotation based on time by news articles presentation in TREC 2005 article publisher. We observe that time-sensitive queries are generally recorded from specific time periods. This statement suggests that it is essential to know the submission of relevant records eventually for a given query. We used the publication duration of the returned records to generate the query regularity histogram. After the generation of the query-frequency histogram our approach is to discover alternate binning techniques based on different underlying theories on how to identify the essential time intervals.

We chosen the best two guideline methods and four time-sensitive methods according to the TREC tests, and omitted the other methods to keep the quantity of individual annotations that we required at controllable stages.

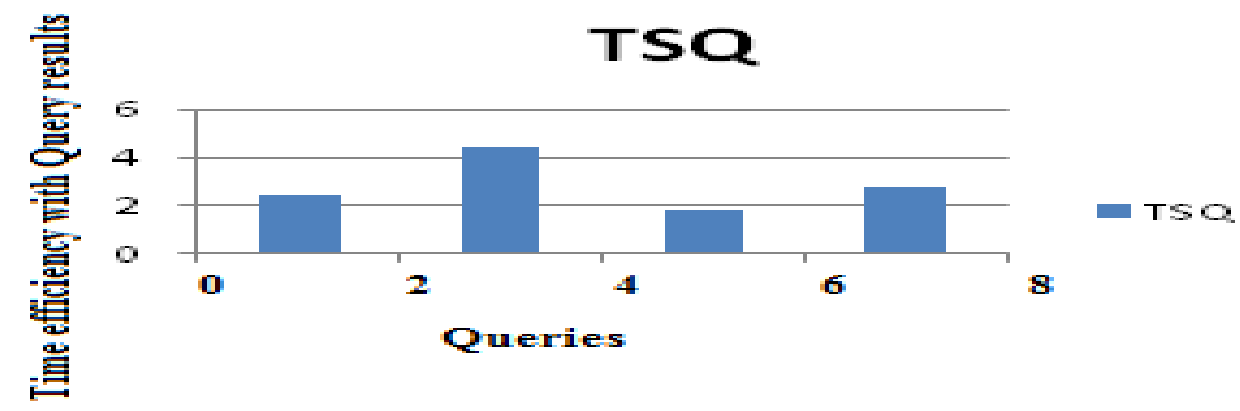

Figure 3. Query retrieval time efficient based on term frequency and inverse document frequency levels.

We have showed that considering time as an additional factor for ranking query results may be valuable for answering time-sensitive queries. Our results indicate that using temporal evidence derived from news archives often increases precision and reveals new relevant documents from important time intervals.

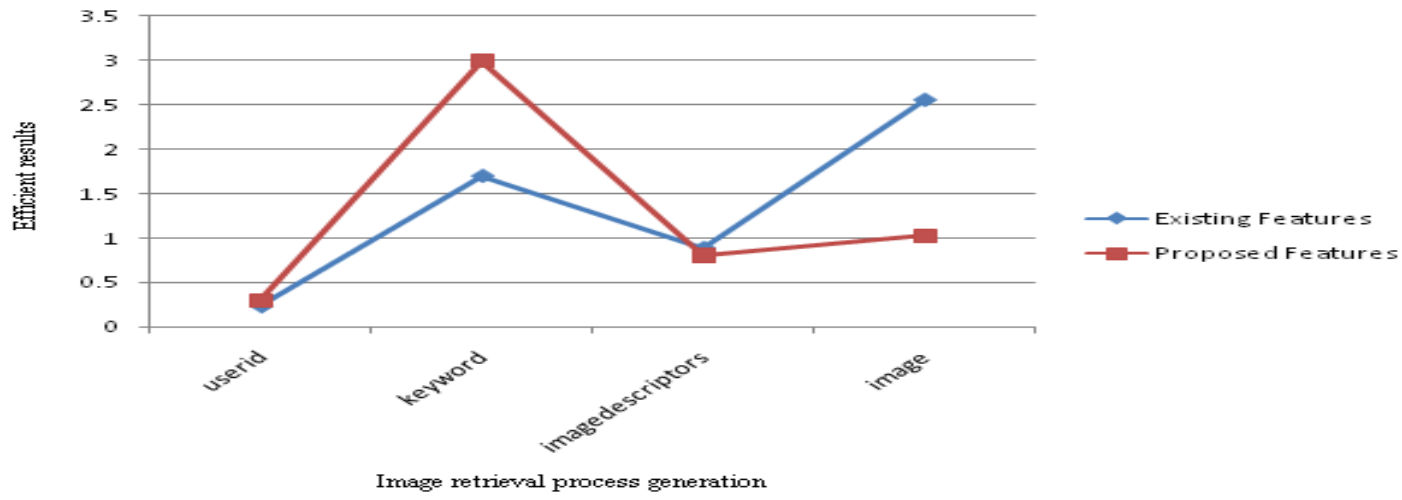

Figure 4. Comparison results with image processing in efficient image retrieval.

In order to assess the efficiency, we used same strategy as we make reference to their evaluation outcomes. For each classification in the 1000 data source images, we arbitrarily chosen 20 images as concerns. For each question, we analyzed the perfection of the recovery based on the importance of the semantic significance between the query image and the recovered images in existing and proposed systems. 


\section{Output Screen shots}

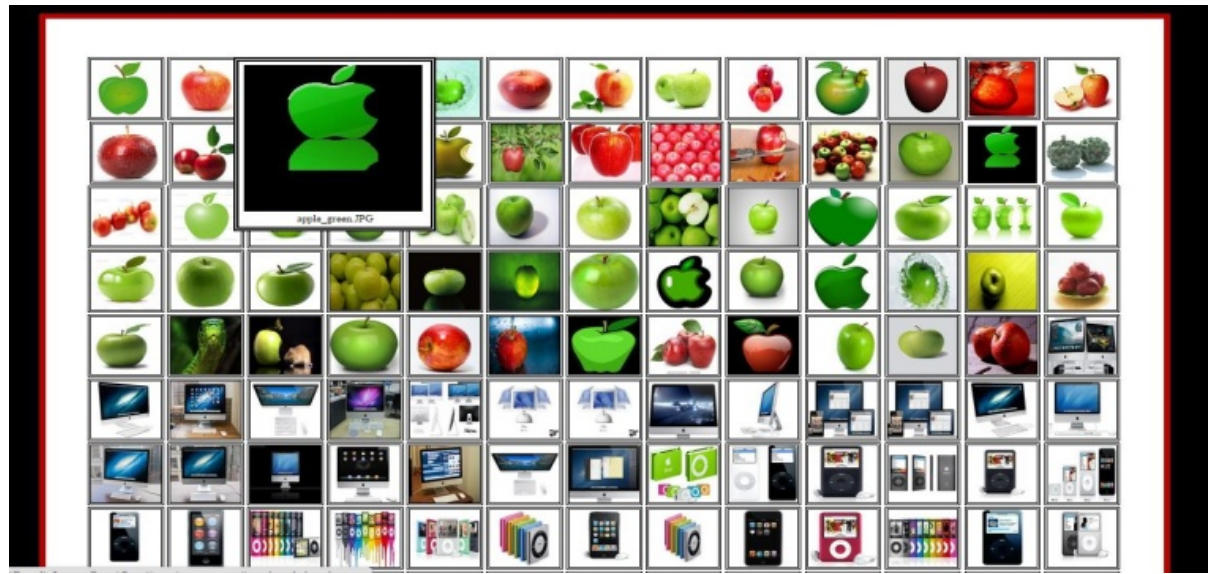

Figure 5. Result based on searching the query word apple

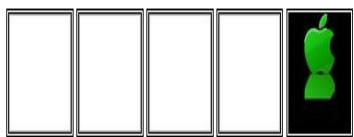

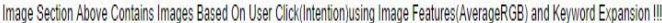
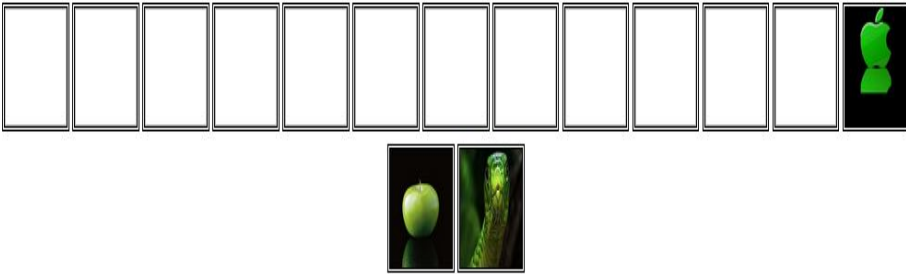

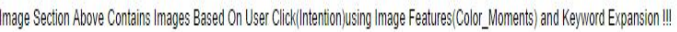

Figure 6. Results based on the features Average RGB and Color Moments

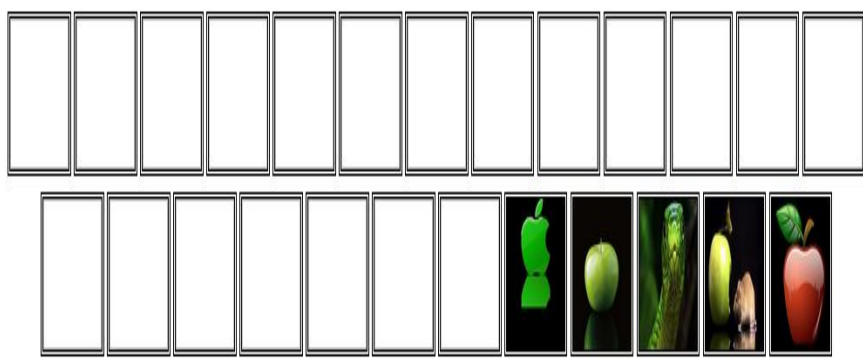

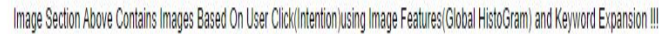

Figure 7. Results based on the feature 


\section{Global Color Histogram}

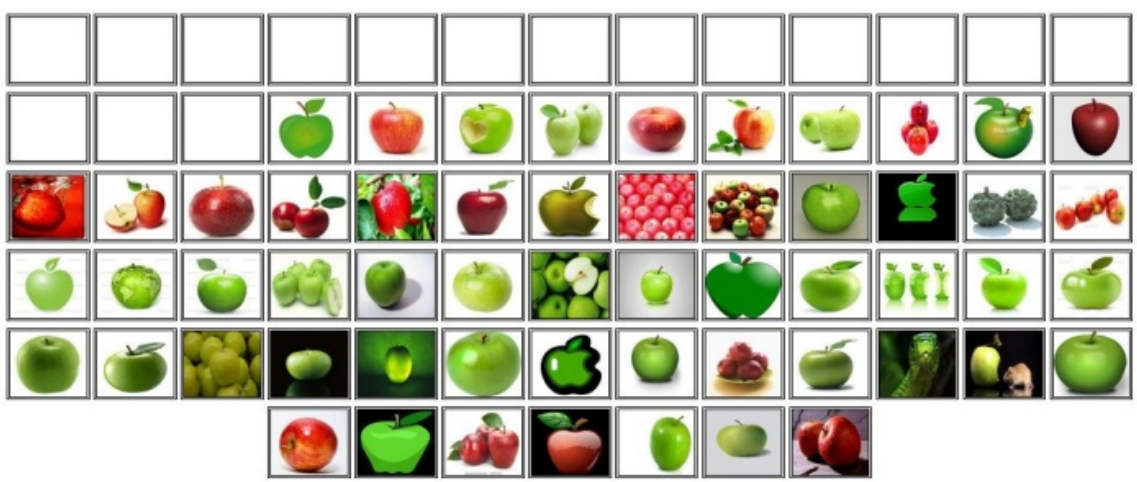

Figure 8. Results based on feature Geometric Moments
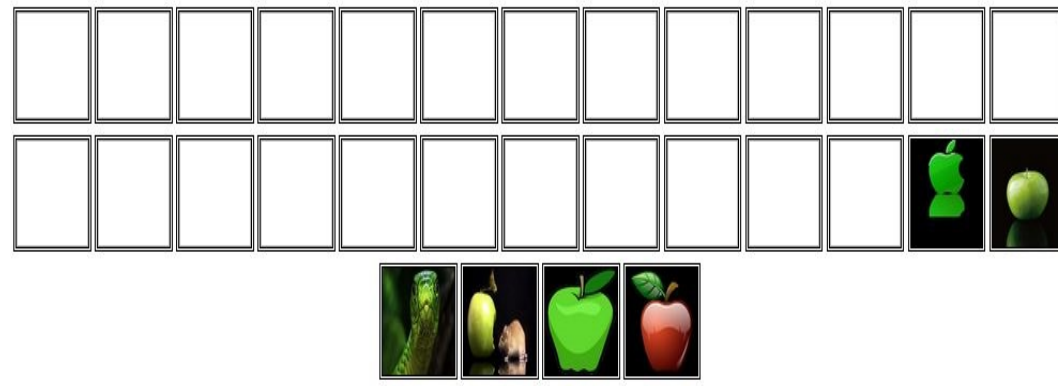

Image Section Above Contains Images Based On User Click(nntention)using Image FeaturesLLocal_Color__istogram) and Keyword Expansion !!I

Figure 9. Results based on feature Local Color Histogram

About $\$ 2$ results acquired in 2.254411962 seconds.

Home

Search Results for Enhancement System

Many Business Books for Sale......good prices

Content Relevance Hits for the keyword(bus): 3 Hits : 1979

Bush 5 WI was Clinton s Wiretapping Initiative

Content Relevance Hits for the keywordous): 2 Published Date 2014-02-10 Hits: 74

Recommendations for a Local BUS Cached IDE Controller_60511

Content Relevance Hits for the keyword(bus): 7

Figure 10. Results based on Document Retrieval Using Query Keyword with publication dates and number of hits 


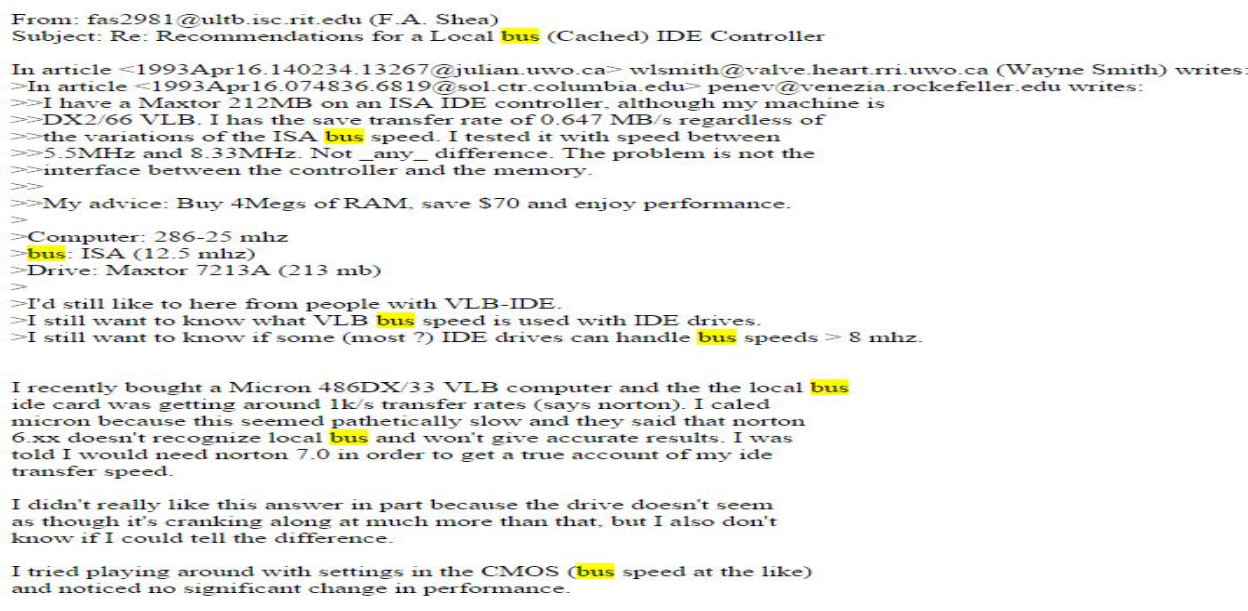

Figure 11. Results showing document retrieval by highlighting query keyword

\section{Conclusion}

In accordance with the keyword and key phrase development and user intension we have to recover appropriate outcomes efficiently. Image retrieval using only text based approach and also using color functions often gives frustrating outcomes, because in many cases, images with identical colors do not have identical material. Content Based Image Retrieval (CBIR) is a set of techniques for accessing semantically-relevant images from an image repository depending on automatically-derived images functions for features like histograms, color moments etc. We provide a comparison between recovery outcomes depending on functions produced from the whole images, and functions produced from images areas. The images are retrieved according to the feature which will be easy for the user for retrieving the exact image and we can also retrieve the relevant documents along with its publication dates and number of hits by using the text based querying technique

\section{References}

[1] S Sunitha, A Rama Satish. "Extended Image Features for User Intention Refined Image Search". in International journal of Advanced Research in Computer Science and Software Engineering. 2014; $4(2)$.

[2] J Cui, F Wen, and X Tang. "Real time google and live image search re-ranking". in Proc. ACM Multimedia. 2008.

[3] "Intentsearch: Interactive on-line image search re-ranking". in Proc. ACM Multimedia. 2008.

[4] N Ben-Haim, B Babenko, and S Belongie. "Improving web-based image search via content based clustering". in Proc. Int'l Workshop on Semantic Learning Applications in Multimedia. 2006.

[5] W Luo, X Wang, and X Tang. "Content-based photo quality assessment". in Proc. Int'I Conf. Computer Vision. 2011.

[6] Y Gangadhar, D Lakshmi Srinivasulu, and VS Giridhar Akula. "Detection and Comparison of Objects Using Two Dimensional Geometric Moment Invariants". International Journal of Information and Education Technology. 2012; 2(5).

[7] Ibrahim SI Abuhaiba1, Ruba AA Salamah. "Efficient Global and Region Content Based Image Retrieval". IJ Image, Graphics and Signal Processing. 2012, 5, 38-46 Published Online June 2012 in MECS (http://www.mecs-press.org/) DOI: 10.5815/ijigsp.2012.05.05.

[8] CD Manning, P Raghavan, and H Schu“ tze. Introduction to Information Retrieval, first ed. Cambridge Univ. Press, July 2008.

[9] "Image Information Retrieval: An Overview of Current Research" by Abby A. Goodrum in, Informing science and special issue on information science research Volume 3 No 22000.

[10] http://vision.ucla.edu/ hbling/publication/emd_pami_O.pdf

[11] http://vis.uky.edu/ cheung/courses/ee639_fall04/readings/EMD.pdf

[12] http://www.cs.cornell.edu/rdz/Papers/ecdl2/spatial.htm

[13] Wynne Hsu, TS Chua, and HK Pung. An integrated color-spatial approach to content-based image retrieval. In ACM Multimedia Conference. 1995: 305--313.

[14] http://antfdez.webs.uvigo.es/publications/2010_AIG_CCR.pdf 
[15] http://citeseerx.ist.psu.edu/viewdoc/download?doi=10.1.1.104.2664\&rep=rep1\&type=pdf

[16] MR Teague. "Image analysis via the general theory of moments". J. Opt. Soc. Amer. Wasington, DC. 1980; 70: 920-930.

JTI Vol. 2, No. 2, September $2014: 57-66$ 